\title{
A novel protodioscin-enriched fenugreek seed extract (Trigonella foenum-graecum, family Fabaceae) improves free testosterone level and sperm profile in healthy volunteers
}

\author{
Anand Swaroop $^{1 *}$, Anuj Maheshwari ${ }^{2,3}$, Narsingh Verma ${ }^{4}$, Kiran Tiwari ${ }^{5}$, Pawan Kumar ${ }^{5}$, \\ Manashi Bagchi ${ }^{1}$, Harry G. Preuss ${ }^{6}$, and Debasis Bagchi ${ }^{1,7}$
}

${ }^{1}$ Cepham Research Center, Piscataway, NJ, USA; ${ }^{2}$ Department of Medicine, BBD University, Lucknow, India; ${ }^{3}$ Metabolic Physician, SHK Diabetes Clinic \& Research Center, Lucknow, India; ${ }^{4}$ Department of Physiology, King George's Medical University, Lucknow, India; ${ }^{5}$ Chemical Resources, Panchkula, Haryana, India; ${ }^{6}$ Georgetown University Medical Center, Washington, DC, USA; ${ }^{7}$ University of Houston College of Pharmacy, Houston, TX, USA

Corresponding Author: Debasis Bagchi, PhD, Pharmacological and Pharmaceutical Sciences, University of Houston College of Pharmacy, Houston, TX 77204, USA

Submission Date: January $23^{\text {rd }}, 2017$, Acceptance Date: April $18^{\text {th }}, 2017$, Publication Date: April $30^{\text {th }}, 2017$

Citation: Swaroop A., Maheshwari A., Verma N., Tiwari K., Kumar P., Bagchi M., Preuss H.G., Bagchi D. A novel protodioscin-enriched fenugreek seed extract (Trigonella foenum-graecum, family Fabaceae) improves free testosterone level and sperm profile in healthy volunteers. Functional Foods in Health and Disease 2017; 7(4); 235-245

\section{ABSTRACT}

Background: A novel, patent-pending 20\% protodioscin-enriched extract has been developed in our laboratories from fenugreek seeds (Furosap ${ }^{\circledR}$ ). We assessed the efficacy of Furosap ${ }^{\circledR}$ in 50 male volunteers (age: 35-65 years) on free and total increased testosterone levels, sperm profile, mental alertness, cardiovascular health, mood, libido, and quality of life.

Methods: Furosap $^{\circledR}(500 \mathrm{mg} /$ day/subject) was administered to the 50 male volunteers over a period of 12 weeks in a one-arm, open-labelled study, to determine the efficacy on free and total testosterone levels, sperm profile and sperm morphology, libido and erectile dysfunction, mood and mental alertness and broad spectrum safety parameters. Institution Review Board approval was obtained for this study and the study was registered at the clinicaltrials.gov (NCT02702882).

Results: A statistically significant increase in free testosterone levels were observed in these volunteers following supplementation of Furosap ${ }^{\circledR}$. Sperm morphology, sperm counts, mental alertness, mood, cardiovascular health, and libido performance were significantly improved. Extensive blood chemistry analyses revealed broad spectrum safety. No significant changes were 
observed in serum lipid function, cholesterol, triglyceride, HDL and LDL levels, and hemogram.

Conclusions: Results confirmed that this protodioscin-enriched extract from fenugreek seeds (Furosap) is safe and efficacious in boosting serum free testosterone levels, healthy sperm profile, mental alertness, cardiovascular health, and overall performance in male volunteers.

Keywords: Standardized fenugreek seed extract; Protodioscin; Testosterone booster; Sperm profile; Mood alleviation; Safety

\section{INTRODUCTION}

Fenugreek plant and seeds are rich in soluble fibers, and extensively used in Ayurvedic, Chinese and Unani medicines for anti-inflammatory, anti-diabetic, antiseptic, aphrodisiac, women's health, and diverse health benefits for centuries [1-9]. Fenugreek leaves, twigs, roots, sprouts, microgreens and yellow to amber colored, cuboid-shaped seeds are extensively used in versatile culinary purposes including spices, salads, soups, brewed into a tea, baked into a bread, and pickles in the Asian countries [1-3, 7-15]. The fenugreek seeds are often roasted to optimize bitterness and flavor [3, 6, 9]. Literature reveals that fenugreek contains approximately 28\% mucilage, $5 \%$ stronger-smelling, bitter fixed oil, rich in phosphates, lecithin and nucleoalbumin, considerable amounts of readily absorbable iron in an organic form, in addition to trigonelline, trimethylamine, choline, biotin, inositol, vitamin A, vitamin B1, vitamin B2, vitamin B3, vitamin B5, vitamin B6, vitamin B9, vitamin B12, and vitamin D, diosgenin, diosgenin- $\beta$-D-glucoside, neurin, betaine, vitexin, vitexin-7-glucoside, yamogenin, vicenin, saponaretin, and isoorientin [4$8,12]$.

Fenugreek and its seeds have exhibited versatile health benefits including antioxidant, antiinflammatory benefits, anti-diabetic, hypercholesterolemia, polycystic ovary syndrome, gastric ulcer and hyperthyroidism, and exercise [4-12]. The dose-dependent efficacy of fenugreek seed extract $(0,150$ or $300 \mathrm{mg} / \mathrm{kg}$ body weight) was investigated over a period of 4 weeks in male mice on endurance capacity in a swimming model [13]. The swimming endurance was significantly increased at $300 \mathrm{mg} / \mathrm{kg}$ body weight dose. The authors reported that the utilization of fatty acids was also significantly increased as an energy source at this dose [13]. Arshadi et al. (2015) also assessed the efficacy of fenugreek seed (0,0.8 or $1.6 \mathrm{~g} / \mathrm{kg}$ body weight) extract in combination with swimming exercise compared to glibenclamide in type 2 diabetic male rats [14, 15]. It was concluded that fenugreek seed consumption, along with swimming exercise, induced a therapeutic efficacy on the improvement of anti-diabetic profiles including plasma insulin, HOMA-IR, plasma leptin, and adiponectin [14, 15].

In a placebo-controlled, double blind study in 49 resistance-trained male volunteers, Poole et al. (2010) assessed the effect of fenugreek supplementation (500 mg/day) on strength, body composition, muscle endurance, power output, and hormonal profiles over a period of 8 weeks. The study demonstrated that fenugreek can significantly increase upper and lower-body strength, reduce body fat and improve overall body composition [16].

This study assessed the efficacy of a novel, patented fenugreek (Trigonella foenum-graecum) seed extract enriched in 20\% protodioscin (Furosap ${ }^{\circledR}$, US Patents\# US 8,217,165 B2; US 8,754, 
205 B2) $[17,18]$ to boost free testosterone levels, sperm profile and morphology, sexual health, sexual arousal, mood and mental alertness, and broad spectrum safety parameters in 50 male volunteers (Age: 35-65 years) over a period of 12 weeks.

\section{MATERIALS AND METHODS}

\section{Trigonella foenum-graecum Seed Extract}

A novel, patented fenugreek (Trigonella foenum-graecum) seed extract (color: off white to light yellow powder, characteristic odor) enriched in $20 \%$ protodioscin (Furosap ${ }^{\circledR}$, Batch\# FUP0814, US Patents\# US 8,217,165 B2; US 8,754, 205 B2) [17, 18]. The powder is $>95 \%$ soluble in water and moisture content is $<5 \%$, both residual solvent and residual pesticide comply with USP3 requirements, and the shelf-life is 2 years.

\section{Ethical Approval}

This study was registered at clinicaltrials.gov (NCT02702882), while the study design, recruitment, and methods were performed in compliance and accordance with the ICH guidelines for Good Clinical Practices (GCP), including the archiving of essential documents, and per international ethical standards guaranteed by the Declaration of Helsinki and its subsequent amendments. Institutional Ethical Board for Medical Research and Institutional Ethics Committee (IEC) from the Ethical Board for Medical Research of Saroj Hospital \& Maternity Center (Kanpur Road, Lucknow, Uttar Pradesh, India) approved this Clinical Study (Reference\# EBMR/2014/07/28/01 dated July 28, 2014). The study was conducted in the Saroj Hospital \& Maternity Center (Kanpur Road, Lucknow, Uttar Pradesh, India). All subjects duly reviewed and signed the Consent Forms. The consent form was submitted with the protocol for review and approved by the IEC. Patient's confidentiality was strictly maintained.

\section{Subject Recruitment and Compliance}

The subjects were systematically screened for the clinical study on the basis of the inclusion/ exclusion criteria as the following. Fifty male subjects were enrolled (age: $43.08 \pm 7.35$ years; body mass index (BMI): $25.46 \pm 4.13 \mathrm{~kg} / \mathrm{m}^{2}$; body weight: $70.38 \pm 12.18 \mathrm{~kg}$; systolic blood pressure $124 \pm 9.40 \mathrm{~mm} \mathrm{Hg}$; diastolic blood pressure $79.65 \pm 6.53 \mathrm{~mm} \mathrm{Hg}$; pulse $77.53 \pm 6.4$ pulse/minute). Inclusion criteria: male subjects (age 35-65 years) diagnosed with symptomatic hypogonadism, understanding the risks/benefits of the protocol, and agreeing to the written in addition to the audio-visual informed consent. Exclusion criteria: uncooperative subjects, receiving any other testosterone boosters for the last 2 months, suffering from coronary artery disease and allied complications, history of malignancy, coagulopathies (clotting and bleeding), psychiatric disorders or hypersensitivity to Furosap ${ }^{\circledR}$, high alcohol intake ( $>2$ drinks per day), abnormal hepatic or renal functions (ALT or AST $>2$ times the upper limit of normal; creatinine $>$ $125 \mu \mathrm{mol} / \mathrm{L}$ ), impaired liver function $>2.5$ times the upper limit of normal, and any particular medical condition where the investigator believes participation in this study could be detrimental to the overall well-being of subjects. Enrolled subjects were given Furosap® (1 capsule of $500 \mathrm{mg}$ each/day after breakfast) over a period of 12 consecutive weeks.

Furosap ${ }^{\circledR}$ was given to the subjects by the site staff and records were maintained in the IP 
accountability log. All data were maintained separately with the date/signature of the principal investigator \& study coordinators. Any concomitant prescription medications taken during study participation were recorded on the case report forms (CRFs). All medications including prescription medications, over-the-counter medications (OTC) and non-prescription medications taken during the clinical study were meticulously recorded and routinely examined by the principal investigator \& study coordinators.

\section{Methods}

The efficacy of Furosap ${ }^{\circledR}(500 \mathrm{mg} /$ day $)$ was assessed on 50 male volunteers, with clinical evaluations being conducted at the baseline, at the end of 4 and 8-weeks, and at the end of 12 weeks. A preliminary in-house study was conducted to determine this effective dose of Furosap ${ }^{\circledR}$. BMI $\left(\mathrm{kg} / \mathrm{m}^{2}\right)$, free testosterone $(\mathrm{pg} / \mathrm{ml})$, total testosterone $(\mathrm{ng} / \mathrm{dl})$, dehydroepiandrosterone sufate (DHEA-S), fasting blood sugar (FBS), fasting lipid profile (total cholesterol, LDL, HDL, triglycerides, VLDL), liver function test (AST, ALT, ALP), semen examination (sperm count, sperm motility, sperm morphology, and hemogram were extensively assessed at the baseline and at the end of 12 weeks respectively. However, BMI, fasting lipid profile and semen examination were assessed at the end of 4 and 8-weeks respectively. Sexual health and sexual arousal were assessed at 4, 8, and 12-weeks of Furosap ${ }^{\circledR}$ treatment.

Free testosterone was measured using a Dia Sources' ELISA kit (catalog\#CAN-FTE-260) purchased from Krishgen Biosystems, Mumbai, India, and total testosterone was assessed using an automated bidirectionally interfaced Chemiluminescent Immunoassay (CLIA) from Siemens Health Care Pvt Ltd, Mumbai, India. Dehydroepiandrosterone sulfate (DHEA-S) was assessed using the Cobas Electrochemiluminescence Immunoassay (ECLIA) (catalog\# 03000087122) kit purchased from Roche Diagnostics India Pvt Ltd, Mumbai, India. Hemoglobin level was evaluated using a Sysmex fully automated bidirectional analyzer (SYSMEX XN-1000) purchased from Transasia Bio Medicals Ltd, Mumbai, India, and fasting blood glucose (FBS) levels were assessed using photometry technology (Agappe Diagnostics Ltd, Mumbai, India). Aspartate Aminotransferase (AST), alkaline phosphatase (ALP), alanine aminotransferase (ALT), blood urea nitrogen (BUN), cholesterol, triglycerides, high density lipoprotein (HDL-C), low density lipoprotein (LDL-C), very low density lipoprotein (VLDL), total leukocytes count (TLC), neutrophils, lymphocytes, monocytes, eosinophils and basophils in Central Processing Lab (CPL, a division of Thyrocare, Mumbai, India) and Regional Processing Lab (RPL, a division of Thyrocare, Mumbai, India). Sperm count, sperm motility and abnormal sperm morphology were assessed in Nigam Pathology (Lucknow, India).

\section{Adverse Events}

Adverse event reporting was strictly enforced.

\section{Statistical Analysis}

Data is expressed as mean \pm SD (standard deviation) or SEM (standard error mean). All parametric and non-parametric assessments were conducted. Wilcoxon signed-rank test, a nonparametric test equivalent to the dependent t-test, was used for assessing mental alertness, mood, reflex erection, and overall performance. 


\section{RESULTS}

\section{Furosap ${ }^{\circledR}(\mathrm{FS})$-induced effect on free testosterone and total testosterone}

Male volunteers were treated with FS over a period of 12 consecutive weeks. Free testosterone and total testosterone levels were assessed at the baseline and at the end of 12 weeks of treatment (Figure 1). Furosap ${ }^{\circledR}$-induced a significant increase in free testosterone level by approximately 1.47 -fold ( $\mathrm{p}$ value $=0.0004^{* *}$ ), while the total testosterone level was increased marginally only by 1.08 -fold ( $\mathrm{p}$-value $=0.164 \mathrm{~ns})$.
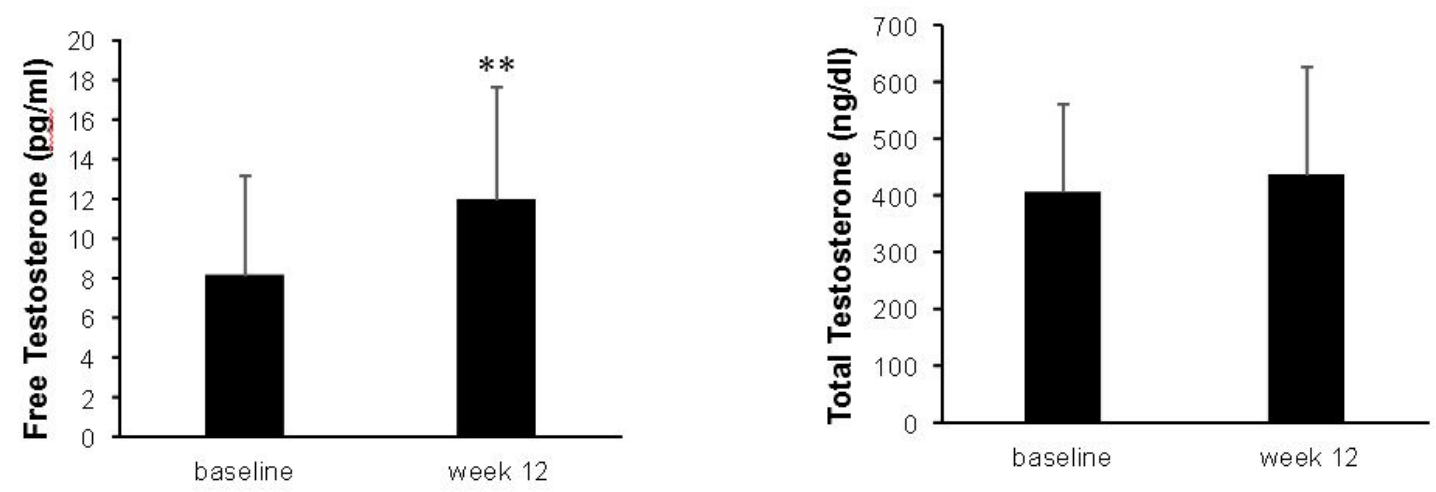

Figure 1. Furosap-induced effect on free and total testosterone at baseline end of 12 weeks of treatment

Effect of Furosap ${ }^{\circledR}$ on sperm count (millions/ml), sperm motility (\%) and abnormal sperm morphology (\%) at baseline, 4-, 8- and 12-weeks of treatment

Sperm count (millions/ml), sperm motility (\%), and abnormal sperm morphology (\%) were evaluated at baseline, after 4 weeks, after 8 weeks and after 12 weeks following supplementation of FS. Sperm count and sperm motility were significantly increased at the end of 4, 8, and 12weeks of FS treatment, while abnormal sperm morphology (\%) reduced at all these time point. Non-significant reduction in abnormal sperm morphology (\%) was recorded at 4-weeks posttreatment. However, abnormal sperm morphology (\%) was significantly reduced both at 8 and 12-weeks post-treatment respectively (Table 1).

Table 1: Effect of Furosap ${ }^{\circledR}$ on sperm count, sperm motility and abnormal sperm morphology at baseline, 4-weeks, 8-weeks, and 12-weeks of treatment

\begin{tabular}{|l|l|l|l|}
\hline Time Point & $\begin{array}{l}\text { Sperm Count } \\
(\mathbf{m i l l i o n s} / \mathbf{m l})\end{array}$ & $\begin{array}{l}\text { Sperm Motility } \\
\mathbf{( \% )}\end{array}$ & $\begin{array}{l}\text { Abnormal Sperm } \\
\text { Morphology (\%) }\end{array}$ \\
\hline Baseline (mean \pm SEM) & $35.13 \pm 2.79$ & $35.79 \pm 2.77$ & $42.46 \pm 2.83$ \\
\hline After 4 weeks (mean \pm SEM) & $48.90 \pm 23.19$ & $45.73 \pm 3.19$ & $39.38 \pm 2.95$ \\
\hline p-value & $0.001^{* *}$ & $0.022^{*}$ & $0.472 \mathrm{~ns}$ \\
\hline Baseline (mean \pm SEM) & $35.13 \pm 2.79$ & $35.79 \pm 2.77$ & $42.46 \pm 2.83$ \\
\hline After 8 weeks (mean \pm SEM) & $86.16 \pm 13.70$ & $35.79 \pm 2.77$ & $21.88 \pm 2.16$ \\
\hline p-value & $0.001^{* *}$ & $0.003^{* *}$ & $0.0003^{* *}$ \\
\hline Baseline $($ Mean \pm SEM) & $35.35 \pm 2.84$ & $35.92 \pm 2.82$ & $42.09 \pm 2.86$ \\
\hline After 12 weeks (mean \pm SEM) & $88.31 \pm 3.18$ & $74.11 \pm 2.13$ & $15.40 \pm 1.61$ \\
\hline p-value & $0.0002^{* *}$ & $0.003^{* *}$ & $0.0002^{* *}$ \\
\hline
\end{tabular}

Values are expressed as mean \pm SEM. $\mathrm{ns}=$ not significant; $* *=$ statistically significant 
Effect of Furosap ${ }^{\circledR}$ on dehydroepiandrosterone sulfate (DHEA-S), fasting blood sugar (FBS) and total leukocyte count (TLC)

DHEA-S, FBS and TLC levels were measured at the baseline and at 12 weeks of treatment. No significant changes were observed (Figure 2).
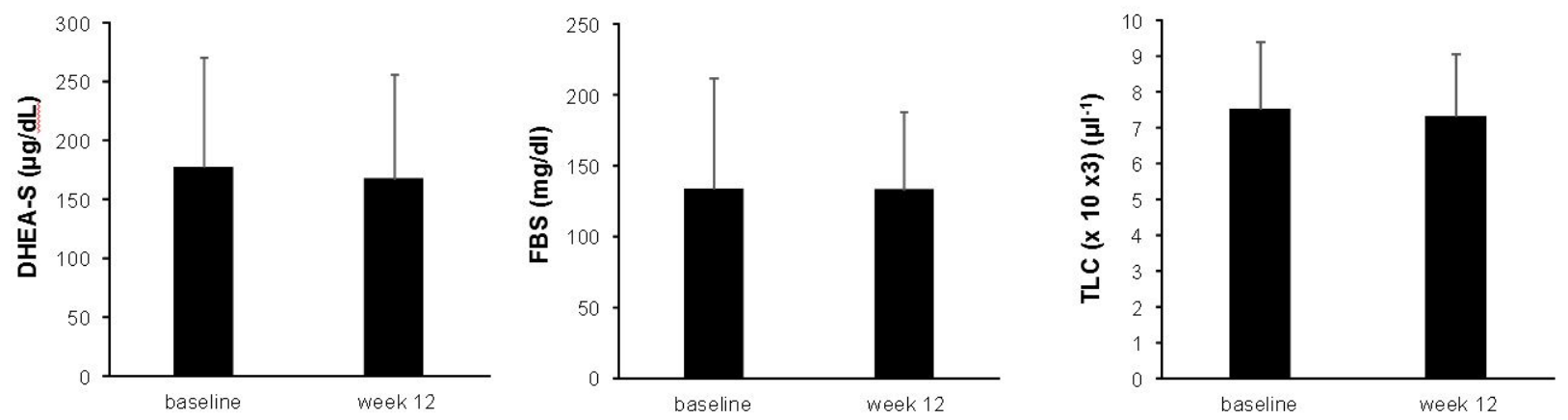

Figure 2. Furosap-induced effect on dehydroeplandrosterone (DHEA-S), fasting blood sugar (FBS) and total leukocyte count (TLC) at baseline end of 12 weeks of treatment

The time-dependent effect of Furosap on mental alertness, mood alleviation, reflex erection and overall performance at the baseline, week 4, week 8, and week 12 of treatment

Mental alertness, mood alleviation, reflex erection, and overall performance were assessed at the baseline, week 4, week 8, and week 12 of treatment (data not shown). Wilcoxon signed-rank test, a nonparametric test equivalent to the dependent t-test, were used to assess the statistical significance. Significant improvements were observed for all these parameters at all time points.

Furosap $^{\circledR}$-induced increases on sexual arousal and excitement at baseline, week 4, week 8, and week 12 of treatment

Subjects demonstrated time-dependent increases in the frequency of sexual arousal (Table 2). Average frequency exhibited a time-dependent escalation, which was approximately doubled at the end of 12 weeks $\left(\mathrm{p}<0.000^{*}\right)$ (Table 2). On visit 1 (after 4-weeks of completion), 65.3\% subjects showed significant improvements, while $95.9 \%$ subjects showed significant improvements after 8-weeks of treatment (visit 2). On visit 3 (after 12-weeks of completion), a total of $98 \%$ population exhibited significant improvement in sexual arousal.

Table 2: Time-dependent efficacy of Furosap ${ }^{\circledR}$ on sexual arousal at baseline, 4-weeks, 8-weeks and 12-weeks of treatment

\begin{tabular}{|l|c|c|}
\hline Time Point & Sexual Arousal & p-value \\
\hline Baseline $($ mean \pm SEM) & $4.02 \pm 1.44$ & $0.000^{* *}$ \\
\hline After 4 weeks $($ mean \pm SEM) & $4.93 \pm 1.25$ & \\
\hline Baseline (mean \pm SEM) & $4.02 \pm 1.44$ & $0.000^{* *}$ \\
\hline After 8 weeks $($ mean \pm SEM) & $6.34 \pm 1.32$ & $0.000^{* *}$ \\
\hline Baseline (Mean \pm SEM) & $4.04 \pm 1.44$ & \\
\hline After 12 weeks (mean \pm SEM) & $8.47 \pm 2.15$ & \\
\hline
\end{tabular}

Values are expressed as mean \pm SEM. $* *=$ statistically significant 


\section{Effect of Furosap ${ }^{\circledR}$ on serum chemistry parameters following supplementation of Furosap ${ }^{\circledR}$} over a period of 12 weeks

No significant changes were observed in serum aspartate aminotransferase/glutamic oxaloacetic transaminase (AST/GOT), alanine aminotransferase/glutamic pyruvic transaminase (ALT/GPT), alkaline phosphatase (ALP), and blood urea nitrogen (BUN) following treatment with Furosap ${ }^{\circledR}$ over a period of 12 weeks.

Time-dependent effect of Furosap ${ }^{\circledR}$ on cholesterol, triglycerides, serum HDL-C, LDL-C and VLDL-C

No significant changes were observed in cholesterol, triglycerides, serum HDL-C, LDL-C, and VLDL-C levels following supplementation of Furosap ${ }^{\circledR}$ over a period of 4, 8, or 12-weeks of treatment

Effects on neutrophils, lymphocytes, monocytes, eosinophils, basophils and hemoglobin levels following supplementation of Furosap ${ }^{\circledR}$

Furosap ${ }^{\circledR}$ didn't induce any significant effects on neutrophils, lymphocytes, monocytes, eosinophils and basophils over a period of 4, 8, or 12-weeks of treatment. A small decrease was observed in the hemoglobin level. However, the baseline and 12-weeks post-treatment hemoglobin levels lied within the normal range.

\section{DISCUSSION}

A broad spectrum of studies has demonstrated that fenugreek (Trigonella foenum-graecum) has exhibited a significant number of health benefits. Fenugreek attenuated body weight gain, obesity and metabolic syndrome related complications, improved insulin sensitivity, anti-diabetic efficacy, muscle building, physical and sexual health, polycystic ovary syndrome, women reproductive disorders, and several others [2, 4, 6-12]. From ancient times, Ayurvedic, Unani, and Chinese medicines have repeated demonstrated the multiple medicinal and therapeutic benefits of fenugreek leaves, twigs, and seeds in a broad spectrum of human diseases and ailments including diabetes, obesity, women reproductive disorders, muscle building, and wrestling [4-7, 19-23]. Fenugreek seeds have demonstrated to contain selected furostanolic saponins, protodioscin, B vitamins, vitamin D, 4-hydroxyisoleucine, diosgenin, diosgenin- $\beta$-D-glucoside, and others. A gas chromatography-mass spectrometry analysis of fenugreek extract revealed the presence of cedrene, eugenol, gingerol, vanillin, and zingerone [24]. An in vitro study by Tomcik et al. [25] demonstrated that fenugreek seeds in combination with insulin significantly modulated creatine content via a mechanism independent of the activity of a sodium and chloride-dependent creatine transporter, SLC6A8 [25]. In the recent past, another independent study by Hamden et al. [26] demonstrated that following the administration of fenugreek seeds to diabetic rats, there was significant decrease of sperm shape abnormality and improvement of the sperm count. Furthermore, the potential protective efficacy of fenugreek seed extract was observed on reproductive systems, as demonstrated by histological studies on testis and epididymis [26]. Aswar et al. [27] assessed the efficacy of fenugreek seed extract $(10 \mathrm{mg} / \mathrm{kg} \mathrm{s.c.} \mathrm{bi-weekly} \mathrm{or} 10$ and $35 \mathrm{mg} / \mathrm{kg}$ body weight orally on immature castrated male Wistar rats. Some anabolic activity 
was observed in these animals without androgenic activity [27].

Fenugreek seed extract also displayed selective cytotoxic activity against T-cell and B-cell lymphoma in vitro. In T-cell lymphoma, the anticancer effect was mediated through induction of apoptosis [28]. Interestingly, protodioscin-enriched fenugreek seed extract exhibited strong growth-inhibitory activity against HL-60 human leukemia cells. Morphological, flow cytometric and molecular analyses revealed induction of apoptosis of tumor cells [11]. Verma et al. [29] reported in vitro anticancer effect of fenugreek whole plant extract against A-549 human lung cancer cells. Treatment of A-549 cells with fenugreek extract diosgenin and pure diosgenin inhibited the growth of cancer cells and caused down-regulation of hTERT expression, indicating abrogation of telomerase activity [30,31]. This may contribute significantly in the application of Furasap $^{\circledR}$ in sports nutrition. Our studies have demonstrated the significant efficacy of Furosap ${ }^{\circledR}$ in significantly improving sperm morphology and down-regulating abnormal sperm morphology.

Multiple human studies were conducted in the recent past demonstrating the efficacy of fenugreek seeds in boosting both free and total testosterone levels, thereby improving sexual and physical health. Testosterone has also been demonstrated to attenuate lean body mass and stronger bones. A clinical investigation in 49 resistance-trained male subjects demonstrated that fenugreek seed extract (500 mg/day) had a significant impact on both upper and lower-body strength in addition to body composition in a double-blind placebo-controlled study [16]. Three independent studies demonstrated the clinical efficacy fenugreek extract in boosting testosterone levels [3234]. The authors indicated that a positive effect was observed on the physiological aspects of libido, muscle strength, and energy. The second study was conducted in 80 healthy menstruating women who reported low sexual drive (age: 20 to 49 years; dose: $600 \mathrm{mg} /$ day), which demonstrated that fenugreek seed extract is beneficial for boosting sexual arousal and desire in women. The third randomized, double-blind, placebo-controlled study was conducted in 120 men (age: 43-70 years; daily dose of fenugreek extract: $600 \mathrm{mg}$ ) over a period of 12 weeks. Both free and total testosterone levels and sexual function increased significantly after 12 weeks of treatment [32-34].

This investigation exhibited that Furosap ${ }^{\circledR}$, enriched in $20 \%$ protodioscin, is instrumental in significantly enhancing free testosterone level, sperm count, sperm motility, mental alertness, mood alleviation, reflex erection, sexual health, sexual arousal, and overall performance in healthy male volunteers.

\section{CONCLUSION}

This clinical study demonstrates that Furosap ${ }^{\circledR}$, a novel, 20\% protodioscin-enriched extract from Trigonella foenum-graecum seeds significantly increased free testosterone levels by 1.47-fold (p value $=0.000^{* *}$ ) following treatment for a period of 12 weeks, while the total testosterone level was marginally increased by 1.08 -fold. Statistically significant increases were observed in sperm count and sperm motility at 4, 8, and 12-weeks of Furosap ${ }^{\circledR}$ treatment, while a statistically significant decrease in abnormal sperm morphology was observed. Sexual health, sexual arousal, and overall performance were significantly increased. Mental alertness, mood alleviation, reflex erection, and overall performance were significantly alleviated at week-4, week-8, and week-12 of treatment. Cardiovascular health was significantly improved. Furthermore, the broad spectrum 


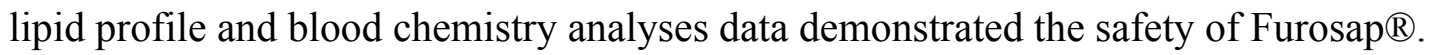

Authors' Contributions \& Conflict of Interest: All authors contributed to this study. AM is the principal investigator and $\mathrm{NV}$ is the co-principal investigator, organized, coordinated the study, and analyzed the data. MB and HGP served as consultants and coordinated in writing the manuscript and coordinated with AM, NV, and DB. DB is the chief scientific officer of Cepham, Inc., and AS is the president of Cepham Inc. KT and PK are involved with the manufacturing and standardization. AM, NV, MB, HGP, and DB have no competing interests.

Funding: The study was funded by Cepham Inc, Piscataway, NJ, USA.

\section{REFERENCES}

1. Basch E, Ulbricht C, Kuo G, Szapary P, Smith M. Therapeutic applications of fenugreek. Altern Med Rev. 2003; 8(1); 20-27.

2. Bahmani M, Shirzad H, Mirhosseini M, Mesripour A, Refieian-Kopaei M. A review of ethnobotanical and therapeutic uses of fenugreek (Trigonella foenum-graecum L.). J Evid Based Complementary Altern Med. 2016; 21(1); 53-62.

3. Yadav UC, Baquer NZ. Pharmacological effects of Trigonella foenum-graecum L. in Health and Disease. Pharm Biol. 2014; 52 (2); 243-254.

4. Roberts KT. The potential of fenugreek (Trigonella foenum-graecum) as a functional food and nutraceutical and its effects on glycemia and lipidemia. J Med Food. 2011; 14(12); 1485-1489.

5. Smith JD, Clinard VB. Natural products for the management of type 2 diabetes mellitus and comorbid conditions. J Am Pharm Assoc. 2014; 54(5); e304-e318.

6. Baquer NZ, Kumar P, Taha A, Kale RK, Cowsik SM, McLean P. Metabolic and molecular action of Trigonella foenum-graecum (fenugreek) and trace metals in experimental diabetic tissues. J Biosci. 2011; 36(2); 383-396.

7. Haber SL, Keonavong J. Fenugreek use in patients with diabetes mellitus. Am J Health Syst Pharm. 2013; 70(14); 1196,1198,1200,1202-1203.

8. Swaroop A, Bagchi M, Kumar P, Preuss HG, Tiwari K, Marone PA, Bagchi D. Safety, efficacy and toxicological evaluation of a novel, patented anti-diabetic extract of Trigonella foenum-graecum Seed Extract (Fenfuro). Toxicol Mech Meth. 2014; 24(7); 495-503.

9. Hua Y, Ren S, Guo R, Rogers O, Bagchi D, Swaroop A, Nair S. Trigonella foenumgraecum seed extract (Fenfuro) inhibits diet-induced insulin resistance and hepatic fat accumulation. Mol Nutr Food Res. 2015; 59(10); 2094-2100.

10. Fuller S, Stephens JM. Diosgenin, 4-hydroxyisoleucine, and fiber from fenugreek: mechanisms of actions and potential effects on metabolic syndrome. Adv Nutr. 2015; 6(2); 189-197.

11. Hibasami H, Moteki H, Ishikawa K, Katsuzaki H, Imai K, Yoshioka K, Ishii Y, Komiya T. Protodioscin isolated from fenugreek (Trigonella foenum-graecum L.) induces cell 
death and morphological change indicative of apoptosis in leukemic cell line HL-60, but not in gastric cancer cell line Kato III. Int J Mol Med. 2003; 11(1); 23-26.

12. Gupta RK, Jain DC, Thakur RS. Minor steroidal sapogenins from fenugreek seeds, Trigonella foenum-graecum J Nat Prod. 1986; 49(6); 1153-1153.

13. Ikeuchi M, Yamaguchi K, Koyama T, Sono Y, Yazawa K. Effects of fenugreek seeds (Trigonella foenum-graecum) extract on endurance capacity in mice. J Nutr Sci Vitaminol (Tokyo). 2006; 52(4); 287-292.

14. Arshadi S, Bakhtiyari S, Haghani K, Valizadeh A. Effects of fenugreek seed extract and swimming endurance training on plasma glucose and cardiac antioxidant enzymes activity in streptozotocin-induced diabetic rats. Osong Public Health Res Perspect. 2015; 6(2); 87-93.

15. Arshadi S, Azarbayjani MA, Hajaghaalipor F, Yusof A, Peeri M, Bakhtiyari S, Stannard RS, Osman NA, Dehghan F. Evaluation of Trigonella foenum-graecum extract in combination with swimming exercise compared to glibenclamide consumption on type 2 diabetic rodents. Food Nutr Res. 2015;59; 29717. doi: 10.3402/fnr.v59.29717. eCollection2015.

16. Poole C, Bushey B, Foster C, Campbell B, Willoughby D, Kreider R, Taylor L, Wilborn C. The effects of a commercially available botanical supplement on strength, body composition, power output, and hormonal profiles in resistance-trained males. J Int Soc Sports Nutr 2010 Oct 27;7; 34. doi: 10.1186/1550-2783-7-34.

17. An extract obtained by a commercially viable process for the extraction of furostanolic saponins from fenugreek seeds, in which one of the compounds in the extract is protodioscin. Inventor: P.K. Goel. United States Patent 8,754,205 B2, Date of Patent Jun 17, 2014.

18. A novel process for the extraction of furostanolic saponins from fenugreek seeds. Inventor: P.K. Goel. United States Patent 8,217,165 B2, Date of Patent Jul 10, 2012.

19. Ulbricht C, Basch E, Burke D, Cheung L, Ernst E, Giese N, Foppa I, Hammerness P, Hashmi S, Kuo G, Miranda M, Mukherjee S, Smith M, Sollars D, Tanquay-Colucci S, Vijayan N, Weissner W. Fenugreek (Trigonella foenum-graecum Leguminosae): An evidence-based systematic review by the natural standard research collaboration. J Herb Pharmacother 2007; 7(3-4); 143-177.

20. Pribaci GC, Sferdian MF, Neamtu C, Craciun C. Fenugreek powder exerts protective effects on alcoholised rats' kidney, highlighted using ultrastructural studies. Rom J Morphol Embryol 2015; 56(2); 445-451.

21. Elujoba AA, Hardman R. Saponin-hydrolyzing enzymes from fenugreek seed. Fitoterapia 1987; 58(3); 197-199.

22. Bordia A, Verma SK, Srivastava KC. Effect of ginger (Zingiber officinale Rosc.) and fenugreek (Trigonella foenum-graceum Linn.) on blood lipids, blood sugar and platelet aggregation in patients with coronary artery disease. Prostaglandins Leukot Essent Fatty Acids 1997; 56(5); 379-384.

23. Jaiswal Y, Liang Z, Zhao Z. Botanical drugs in Ayurveda and traditional Chinese medicine. J Ethnopharmacol. 2016; 194; 245-259. 
24. Al-Daghri NM, Alkoil MS, Alkharfy KM, Mohammed AK, Abd-Alrahman SH, Yakout SM, Amer OE, Krishnaswamy S. Fenugreek extract as an inducer of cellular death via autophagy in human T lymphoma Jurkat cells. BMC Complement Altern Med. 2012 Oct 30;12:202. doi: 10.1186/1472-6882-12-202.

25. Tomcik KA, Smiles WJ, Camera DM, Hugel HM, Hawley JA, Watts R. Fenugreek increases insulin-stimulated creatine content in L6C11 muscle myotubes. Eur J Nutr. 2016 Jan 5. [Epub ahead of print].

26. Hamden K, Jaouadi B, Carreau S, Aouidet A, El-Fazaa S, Gharbi N, Effeki A. Potential protective effect on key steroidogenesis and metabolic enzymes and sperm abnormalities by fenugreek steroids in testis and epididymis of surviving diabetic rats. Arch Physiol Biochem. 2010; 116(3); 146-155.

27. Aswar U, Bodhankar SL, Mohan V, Thakurdesai PA. Effect of furostanol glycosides from Trigonella foenum-graecum on the reproductive system of male albino rats. Phytother Res. 2010; 24(10); 1482-1488.

28. Wankhede S, Mohan V, Thakurdesai P. Beneficial effects of fenugreek glycoside supplementation in male subjects during resistance training: A randomized controlled pilot study. J Sport Health Sci. 2016; 5(2); 176-182.

29. Verma SK, Singh SK, Mathur A. In vitro cytotoxicity of Calotropis procera and Trigonella foenum-graecum against human cancer cell lines. J Chem Pharm Res. 2010; 2(2); 861-165.

30. Rahmati-Yamchi M, Ghareghomi S, Haddadchi G, Milani M, Aghazadeh M, Daroushnejad H. Fenugreek extract diosgenin and pure diosgenin inhibit the hTERT gene expression in A549 lung cancer cell line. Mol Biol Rep. 2014; 41(9); 6247-6252.

31. Rahmati-Yamchi M, Ghareghomi S, Haddadchi G, Majid M, Yousef R. Diosgenin inhibits hTERT gene expression in the A 549 lung cancer cell line. Asian Pac J Cancer Prev. 2013; 14(3); 6945-6948.

32. Rao A, Steels E, Inder WJ, Abraham S, Vietta L. Testofen, a specialized Trigonella foenum-graecum seed extract reduces age-related symptoms of androgen decrease, increases testosterone levels and improves sexual function in healthy aging males in a double-blind randomised clinical study. Aging Male. 2016; 19(2); 134142.

33. Rao A, Steels E, Beccaria G, Inder WJ, Vietta L. Influence of a specialized Trigonella foenum-graecum seed extract (libifem), on testosterone, estradiol and sexual function in healthy menstruating women, a randomised placebo controlled study. Phytother Res. 2015; 29(8); 1123-1130.

34. Steels E, Rao A, Vietta L. Physiological aspects of male libido enhanced by standardized Trigonella foenum-graecum extract and mineral formulation. Phytother Res. 2011; 25(9); 1294-1300. 